\title{
Excision of singularities by stringy domain walls
}

Renata Kallosh

Physics Department, Stanford University, Stanford, California 94305

Thomas Mohaupt

Physics Department, Stanford University, Stanford, California 94305

and Fachgruppe Theoretische Physik, Universität Halle, D-06099 Halle

Marina Shmakova

California Institute for Physics and Astrophysics 366 Cambridge Avenue, Palo Alto, California 94306

(Received 2 January 2001; accepted for publication 13 February 2001)

\begin{abstract}
We study supersymmetric domain walls on $S_{1} / \mathbf{Z}_{2}$ orbifolds. The supergravity solutions in the bulk are given by the attractor equation associated with Calabi-Yau (CY) spaces and have a naked space-time singularity at some $\left|y_{s}\right|$. We are looking for possibilities to cut off this singularity with the second wall by a stringy mechanism. We use the collapse of the CY cycle at $\left|y_{c}\right|$ which happens before and at a finite distance from the space-time singularity. In our example with three Kähler moduli the second wall is at the end of the moduli space at $\left|y_{c}\right|$ where also the enhancement of $\mathrm{SU}(2)$ gauge symmetry takes place so that $\left|y_{e}\right|=\left|y_{c}\right|<\left|y_{s}\right|$. The physics of the excision of a naked singularity via the enhançon in the context of domain wall has an interpretation on the heterotic side related to $R \rightarrow 1 / R$ duality. The position of the enhançon is given by the equation $R\left(\left|y_{e}\right|\right)=1$. (C) 2001 American Institute of Physics. [DOI: 10.1063/1.1373424]
\end{abstract}

The supersymmetric domain wall solutions of $D=5, N=2, \mathrm{U}(1)$ gauged supergravity ${ }^{1}$ with brane sources on $S^{1} / \mathbf{Z}_{2}$ orbifolds have been described recently in Ref. 2 . It has been observed there that in the context of Calabi-Yau (CY) compactifications the collapse of CY cycles may put some restrictions on the distance between the walls. ${ }^{3,4}$ In this article we will study this type of domain wall both for $D=5, N=2$, U(1) gauged supergravity ${ }^{1}$ (GST model) and for Calabi-Yau compactifications of 11-D supergravity with fluxes turned on. The latter is the five-dimensional heterotic M-theory ${ }^{5,6}$ obtained by a reduction on a CY threefold of Horava-Witten M-theory ${ }^{7}$ on $S^{1} / \mathbf{Z}_{2}$ (HW model). The explicit form of the solution with general dependence on the vector multiplets is obtained for both models by solving the generalized attractor equation. ${ }^{8-11}$ Since the domain wall solutions ${ }^{2,6}$ of the two models behave very similarly, we will discuss them in parallel.

The purpose of this article is to find a possibility to remove the space-time singularity of the domain wall solution via some particular property of the CY space. Specifically we would like to find a situation when the collapse of the CY cycle at $\left|y_{c}\right|$ happens closer to the first wall which is at $y=0$ and at a finite distance from the space-time singularity $\left|y_{s}\right|$, so that

$$
\left|y_{c}\right|<\left|y_{s}\right|
$$

In the case of excision of repulson singularities by the enhançon mechanism, ${ }^{12}$ the distance between the repulson and enhançon is finite. The hope, therefore, is that also for some domain walls the analogous situation may be possible, particularly if enhancement of gauge symmetry is somehow involved. The finite distance between the naked singularity of the supergravity solution and the position of the collapse of the CY cycle may allow us to use the physics of string theory already at the end of the moduli space which in this case excludes the singularity of the general relativity as unphysical. The generic interest in such a mechanism is supported by some interesting 
recent investigations of the brane world scenarios ${ }^{13}$ where the naked singularities may be present in solutions of the Einstein equations and need to be removed by a natural stringy-type mechanism.

Our walls are supersymmetric everywhere, including the position of the branes, ${ }^{2}$ therefore they do not directly address the problems of the models in Ref. 13. But due to supersymmetry in our model the matching conditions for the solutions are satisfied automatically on both walls. We have more control over the situation and may clearly indicate conditions when a natural mechanism of stringy excision of singularities is available.

We have found that in most cases the singularities of the CY space tend to coincide with the space-time singularity of the domain wall solutions, i.e.,

$$
\left|y_{c}\right|=\left|y_{s}\right| .
$$

Only in some special cases we will find the singularities in space-time and CY space at some finite distance in the $y$ direction from each other as in Eq. (1). Within the classification of the possible behavior of the CY manifold at the boundary of the Kähler cone $e^{9,14}$ we consider the special case when a complex divisor $D$ is collapsing to a curve $E$ of $A_{1}$ singularities, so that there is an $\mathrm{SU}(2)$ enhancement of gauge symmetry at the boundary. The position $\left|y_{c}\right|$ where the cycle collapses therefore corresponds to the position $\left|y_{e}\right|$ of the enhançon. We will find examples where

$$
\left|y_{c}\right|=\left|y_{e}\right|<\left|y_{s}\right| .
$$

Domain wall solutions of the two models have a metric of the form ${ }^{15}$

$$
d s^{2}=a^{2}(y) d x^{\mu} d x^{\nu} \eta_{\mu \nu}+a^{2 \gamma}(y) d y^{2},
$$

where $\gamma=-2$ is the GST model and $\gamma=4$ is the HW model. ${ }^{16}$ In both cases the function $a(y)$ and the scalar fields are determined in terms of harmonic functions through generalized attractor equations ${ }^{9,10}$ which require that the rescaled variables $\widetilde{h}^{I} \equiv a(y) h^{I}$ have to satisfy

$$
C_{I J K} \widetilde{h}^{J} \widetilde{h}^{k}=H_{I}(y),
$$

where $H_{I}(y)$ are harmonic functions. Then the physical scalars are given by either solving the hypersurface constraint or by using the the ratios $\widetilde{h}^{x} / \widetilde{h}^{0}$ and the metric is determined by

$$
a^{3}(y)=C_{I J K} \widetilde{h}^{I} \widetilde{h}^{J} \widetilde{h}^{K}=\widetilde{h}^{I} H_{I} .
$$

In the HW model one additional scalar enters nontrivially into the solution. This scalar is the overall volume $V$, or "breathing mode" of the Calabi-Yau space. Since there is no solution with constant $V$, there are no anti-de Sitter (AdS) vacua in the HW case in contrast to domain walls in 5-D supergravity. ${ }^{2}$ For our purpose it is important that $V$ is uniquely determined by the vector multiplet scalars. In fact, $V$ is just some power of $a$, and therefore a rational function of the harmonic functions:

$$
V=a^{6}=\left(C_{I J K} \widetilde{h}^{I} \widetilde{h}^{J} \widetilde{h}^{K}\right)^{2} .
$$

As a consequence the flow through moduli space is the same as in the GST model. The two models differ in the precise form of the space-time metric and by the fact that $a^{6}$ in the HW case is the volume of the internal space.

Following Ref. 2 we consider a setup where the fifth direction is a $S^{1} / \mathbf{Z}_{2}$ orbifold with three-branes at the fixed points $y=0$ and $y=\tilde{y}$, which act as sources for the harmonic functions:

$$
\partial_{y}^{2} H_{I}=-2 g q_{I}[\delta(y)-\delta(y-\widetilde{y})], \quad H_{I}=c_{I}-2 g q_{I}|y| .
$$


Concerning space-time singularities both models behave very similarly. Components of curvature tensors become singular if either $a=0$ or if its derivatives diverge. The corresponding Ricci scalar is

$$
\mathcal{R}=-4 a^{-2-2 \gamma}\left((3-2 \gamma)\left(a^{\prime}\right)^{2}+2 a a^{\prime \prime}\right)
$$

To find explicit domain wall solutions we consider some CY spaces with three Kähler moduli ${ }^{17,18}$ for which the relevant prepotential was identified in 5-D supergravity and the attractor equations have been solved. Many of such solutions are displayed and analyzed in Refs. 9 and 11 for the extended Kähler cone of a CY which is an elliptic fibration over the Hirzebruch surface $\mathcal{F}_{1}$. The extended Kähler cone consists of two Kähler cones related by a flop transition. ${ }^{19}$ We will refer to the two CY compactifications as model III and model II, respectively. Model I forms a particular boundary of the moduli space of the model II. The moduli space of model III has a boundary where SU(2) enhancement occurs in the way described above. Moreover, the metric on the moduli space is finite at this boundary. As explained in Refs. 17 and 18, the region III CY is related to two other CY spaces by deformation of the base of the elliptic fibration into the Hirzebruch surfaces $\mathcal{F}_{0}$ and $\mathcal{F}_{2}$, respectively. These models likewise have a boundary with $\mathrm{SU}(2)$ enhancement, and the physics close to the boundary is completely isomorphic to the one of the region III $\mathcal{F}_{1}$ model. Though we will discuss the region III model for definiteness, our results will be automatically valid for these models as well. The M-theory compactifications on the elliptically fibered CY spaces with bases $\mathcal{F}_{0}, \mathcal{F}_{1}, \mathcal{F}_{2}$ have a dual description by compactifications of the $E_{8} \times E_{8}$ heterotic string on $K 3 \times S^{1}$ with instanton numbers $(12,12),(13,11),(14,10)$, respectively. To be precise this duality is known to be valid in the absence of $G$-flux inside the M-theory CY. We will later use the heterotic picture to describe the $\mathrm{SU}(2)$ enhancement in a simple way, assuming that the duality is still valid in presence of $G$-flux. Since switching on $G$-flux does not interfere with the mechanism underlying gauge symmetry enhancement this is a reasonable assumption.

Let us return to the M-theory compactification on the CY with base $\mathcal{F}_{1}$. The boundaries of the extended Kähler cone are defined by the collapse of some cycles to zero volume. The whole picture is shown in Fig. 2 of Ref. 9. Some of the boundaries have a vanishing metric of the moduli space, some have an infinite metric. Equations of motion relate the space-time curvature $\mathcal{R}$ with expressions which depend on moduli space metric, $g_{x y}\left(\phi^{x}\right)^{\prime}\left(\phi^{y}\right)^{\prime} a^{-2 \gamma}$, as well as with expressions depending on the inverse moduli space metric, $W_{, x} g^{x y} W_{, y}$. This indicates that it is likely that the space-time curvature is infinite if the moduli space metric $g_{x y}$ or its inverse $g^{x y}$ are infinite. ${ }^{20}$ We have studied several cases explicitly and found that they indeed have coinciding singularities of the space-time and the moduli space.

We proceed therefore directly with the domain walls of the model III, which has a boundary with finite metric. The classical prepotential for this model was derived in Ref. 18. In terms of the variables adapted to the Kähler cone, the prepotential is

$$
\mathcal{V}=\frac{4}{3}\left(h^{1}\right)^{3}+\frac{3}{2}\left(h^{1}\right)^{2} h^{2}+\frac{1}{2} h^{1}\left(h^{2}\right)^{2}+\left(h^{1}\right)^{2} h^{3}+h^{1} h^{2} h^{3}=1,
$$

and the Kähler cone is simply $h^{I}>0$. In the new variables

$$
h^{1}=U, \quad h^{2}=T-\frac{1}{2} U-W, \quad h^{3}=W-U,
$$

the prepotential becomes

$$
\mathcal{V}=\frac{5}{24} U^{3}+\frac{1}{2} U T^{2}-\frac{1}{2} U W^{2}+\frac{1}{2} U^{2} W=1 .
$$

The Kähler cone is $W>U>0$ and $T>W+1 / 2 U$. There are three boundaries when either of $h^{I}$ vanishes: (i) $U=0 \Leftrightarrow h^{1}=0$ : here the metric of moduli space becomes singular, (ii) $T=W$ $+U / 2 \Leftrightarrow h^{2}=0$ : the metric of moduli space is regular and one has non-Abelian gauge symmetry enhancement; and (iii) $W=U \Leftrightarrow h^{3}=0$ : there is a flop transition, and again the metric is regular. We can solve (12) for $T: T=\sqrt{(2 / U)\left(1-5 U^{3} / 24-U^{2} W / 2+U W^{2} / 2\right)}$ and keep as independent 
scalars $\phi^{x}=(U, W)$. By looking at the resulting moduli space metric $g_{x y}$ with determinant $\operatorname{det} g_{x y} \simeq 12\left(3-4 U^{3}\right) /\left(24 U-5 U^{4}-12 U^{3} W+12 U^{2} W^{2}\right)$ we recover the picture given in Fig. 2 in Ref. 9: $U$ varies within a finite interval, whereas $W$ varies from 0 to $\infty$ at $U=0$ and is cutoff by the curves $U=W$ and $T-W-1 / 2 U$ for positive $W$ :

$$
0<U<\left(\frac{3}{4}\right)^{1 / 3}, \quad U<W<\frac{3-U^{3}}{3 U^{2}} .
$$

The stabilization equations (5) are a system of quadratic equations for the rescaled scalars $\widetilde{h}^{I}$ $=a(y) h^{I}:=\widetilde{U}, \widetilde{T}, \widetilde{W}$. For our model they are solved following Ref. 9 by ${ }^{21}$

$$
\widetilde{U}=\sqrt{\alpha-\sqrt{\alpha^{2}-\beta}}, \quad \widetilde{T}=\frac{H_{T}}{\widetilde{U}}, \quad \widetilde{W}=\frac{1}{2} \widetilde{U}-\frac{H_{W}}{2 \widetilde{U}},
$$

where

$$
\alpha=\frac{1}{4}\left(H_{U}+\frac{1}{2} H_{W}\right), \quad \beta=\frac{1}{8}\left(H_{T}^{2}-H_{W}^{2}\right) .
$$

One needs to impose that the scalars are real and inside the Kähler cone. Therefore the harmonic functions are subject to the inequalities

$$
\frac{2}{3} H_{U} \geqslant H_{T} \geqslant-H_{W} \geqslant \frac{2}{9}\left(H_{U}-\sqrt{H_{U}^{2}-\frac{9}{4} H_{T}^{2}}\right),
$$

which are mutually consistent. The boundary $T=W+U / 2$ corresponds to $2 H_{U}=3 H_{T}$, whereas the boundary $W=U$ corresponds to $-H_{W}=2 / 9\left(H_{U}-\sqrt{H_{U}^{2}-9 / 4 H_{T}^{2}}\right)$ and the boundary $U=0$ corresponds to $H_{T}=-H_{W}$. We would like to mention that the second branch of the attractor equations found in Ref. 11 does not describe a solution inside the Kähler cone, as can be verified by a full analysis of the constraints.

Let us show that for generic values of the parameters of the harmonic functions the collapse of the modulus $h^{2}$ is taking place at the point $\left|y_{c}\right|$ which is at a finite distance from the space-time singularity.

First of all we have to find out under which conditions space-time curvature can diverge. Looking at the formula (9) for the Ricci scalar we find that this happens if either $a=0$ or one of its derivatives diverges. ${ }^{22}$ The same is true for the components of the Ricci tensor and of the Riemann tensor, which we did not display explicitly. The only point within the extended Kähler cone where $a$ vanishes is $U=W=0, T=\infty$. At this point the moduli space metric is infinite. Divergences in the derivatives of $a$ occur when either $\alpha=\sqrt{\alpha^{2}-\beta}$ or $\alpha^{2}=\beta$. The first case corresponds to $U=0$, which is a boundary of the Kähler cone on which the moduli space metric diverges. This includes the point where $a=0$ as a subcase. Thus on the boundary $U=0$ one finds the expected coincidence of space-time singularities with moduli space singularities. The only kind of space-time singularities which need to concern us here are the ones related to $\alpha^{2}=\beta$.

The equation $\alpha^{2}=\beta$ has no solutions if $9 H_{T}^{2}<4 H_{U}^{2}$ which corresponds to $T>W+1 / 2 U$. Therefore no space-time singularity can occur as long as the moduli are inside the Kähler cone. If $9 H_{T}^{2}>4 H_{U}^{2}$, then $\alpha^{2}=\beta$ has two solutions, $H_{W}=-2 / 9\left(H_{U} \pm \sqrt{8} \sqrt{9 / 4 H_{T}^{2}-H_{U}^{2}}\right)$. Thus the generic situation is that one first crosses the enhancement boundary $T=W+1 / 2 U$ and then runs into a space-time singularity at a finite distance. If $9 H_{T}^{2}=4 H_{U}^{2}$, which is precisely true on the enhancement boundary, then $\alpha^{2}=\beta$ has one solution given by $2 H_{U}+9 H_{W}=0$. Thus the only possibility for the space-time singularity to coincide with the boundary of moduli space is when the parameters are fine tuned such that $2 H_{U}\left(y_{c}\right)=3 H_{T}\left(y_{c}\right)=-9 H_{W}\left(y_{c}\right)$. The corresponding point in moduli space is the intersection point of the enhancement boundary $T=W+1 / 2 U$ with the flop boundary $U=W$. At this point the metric on moduli space is degenerate, which nicely fits with our observation that a singularity in moduli space generically induces a singularity in space-time. 
The coordinates $W, T, U$ cover both regions of model III and II and allow us to analyze the flop transition in the framework of special geometry, as shown in Ref. 9. It is also interesting to use the description of the region III in STU parametrization. In this parametrization we can also show that the singularities of the space-time and CY space are at finite distance and we will give a numerical example. Moreover, the interpretation of the enhançon-type physics in terms of T-duality is manifest.

After the substitution $W=S^{\prime}-1 / 2\left(T^{\prime}-U^{\prime}\right), T=S^{\prime}+1 / 2 T^{\prime}$, and $U=U^{\prime}$, the region III prepotential takes the form

$$
\mathcal{V}=S^{\prime} T^{\prime} U^{\prime}+\frac{1}{3} U^{\prime 3}
$$

The original CY Kähler moduli are now related to the heterotic string variables as follows:

$$
h^{1}=U^{\prime}, \quad h^{2}=T^{\prime}-U^{\prime}, \quad h^{3}=S^{\prime}-\frac{1}{2}\left(T^{\prime}+U^{\prime}\right) .
$$

The solution in these variables is ${ }^{23}$

$$
\tilde{U}^{\prime}=\frac{1}{2} \sqrt{H_{U}^{\prime}-\sqrt{\left(H_{U}^{\prime}\right)^{2}-4 H_{S}^{\prime} H_{T}^{\prime}}}, \quad \widetilde{T}^{\prime}=\frac{H_{S}^{\prime}}{2 \widetilde{U}^{\prime}}, \quad \tilde{S}^{\prime}=\frac{H_{T}^{\prime}}{2 U^{\prime}}
$$

Note that the harmonic functions are now associated with the primed variables. The boundaries of region III are

$$
\begin{gathered}
U>0 \Leftrightarrow U^{\prime}>0, \\
W>U \Leftrightarrow S^{\prime}>\frac{1}{2}\left(T^{\prime}+U^{\prime}\right), \\
T>W+\frac{U}{2} \Leftrightarrow T^{\prime}>U^{\prime} .
\end{gathered}
$$

For convenience we drop the primes on moduli and harmonic functions in the rest of the article, denoting moduli simply by $S, T, U$. One should keep in mind that the $T$-variables in both parametrizations are different!

Let us look at the moduli space metric. We solve the hypersurface equation $\mathcal{V}=S T U$ $+1 / 3 U^{3}=1$ for $S: S=\left(3-U^{3}\right) / 3 T U$. The determinant of the vector kinetic matrix is $\operatorname{det} G_{I J} \simeq 1$ $-4 / 3 U^{3}$. Thus $0 \leqslant U \leqslant(3 / 4)^{1 / 3}$ as expected, because the $U$ variable is the same in the $T U W$ and $S T U$ parametrization. The determinant of the scalar kinetic term is

$$
\operatorname{det} g_{x y} \simeq \frac{3-4 U^{3}}{T^{2} U^{2}}
$$

implying $0<U<(3 / 4)^{1 / 3}$ and $T \neq 0$. Since $T$ is positive for our CY moduli space, the moduli space metric is regular for

$$
0<U<\left(\frac{3}{4}\right)^{1 / 3} \text { and } \quad 0<T
$$

In particular, it becomes singular on $U=0$, which is a boundary from the CY point of view (tensionless strings). On the boundary $U=T$ (symmetry enhancement) it is regular, as long as $U$ takes allowed values. The third boundary (flop) is given by

$$
S(T, U)=\frac{1}{2}(T+U)
$$


which can be solved for $T$ as $T(U)=-1 / 2 U+(\sqrt{3} / 6 U) \sqrt{24 U-5 U^{4}}$. Note that $T(U)$ is positive for all $0<U<(3 / 4)^{1 / 3}$. Therefore the moduli space metric is regular along the flop line. The reality of the "inner" and "outer" roots in (19) imposes

$$
H_{U}^{2}>4 H_{S} H_{T}>0 \text {. }
$$

A further look at (19) and (20) tells us that the harmonic functions $H_{S}, H_{T}, H_{U}$ have to be positive. When combining this with (24) then all expressions are real and $U>0$. The other boundaries are $T \geqslant U$ and $S \geqslant 1 / 2(T+U)$. The condition $T \geqslant U$ takes a very simple form,

$$
H_{U} \geqslant H_{S}+H_{T},
$$

which is compatible with (24). We are interested in the limit $H_{U} \rightarrow H_{S}+H_{T}$. We still have to implement the constraint that $h^{3}$ is positive, which in these variables is $S>1 / 2(T+U)$. We will impose the stronger constraint $S>T$ which yields a simpler constraint on the harmonic functions and has the additional advantage to guarantee that our solution is also inside the Kähler cones of the related $\mathcal{F}_{0}$ and $\mathcal{F}_{2}$ models. For these models the prepotential likewise can be brought to the form (17). However, the boundaries of the Kähler cones are different. For the $\mathcal{F}_{2}$ model the Kähler cone is defined by $S>T>U>0$, whereas for the $\mathcal{F}_{0}$ model one has $S, T>U>0$. Note that all models share the $U=T$ boundary, though the other boundaries are different. Moreover, when imposing the strongest constraint $S>T>U>0$ we can discuss the limit $T-U \rightarrow 0$ for all three models simultaneously. Now $S>T$ simply implies

$$
H_{T}>H_{S} .
$$

The constraints we found are compatible: evaluating (24), when (26) is saturated, gives $\left(H_{T}\right.$ $\left.-H_{S}\right)^{2} \geqslant 0$. Thus the boundary $U=T$ requires that $H_{U}=H_{S}+H_{T}$. This defines its position as

$$
\left|y_{c}\right|=\frac{c_{U}-c_{T}-c_{S}}{d_{U}-d_{T}-d_{S}} .
$$

A closer inspection of the analytic form of the Ricci scalar $\mathcal{R}$ and of the function $a$ and its space-time derivatives shows that curvature singularity precisely occurs when $H_{U}^{2}=4 H_{S} H_{T}$. Given the inequalities (24)-(26) we see that this can never happen inside the Kähler cone. Moreover, the generic situation is that the space-time singularity is encountered after crossing the $T$ $=U$ boundary. The only possibility to have the space-time singularity coincide with the boundary of moduli space is to fine tune the parameters such that $H_{T}\left(y_{c}\right)=H_{S}\left(y_{c}\right)$ coincides with $H_{U}^{2}\left(y_{s}\right)=4 H_{T}\left(y_{s}\right) H_{U}\left(y_{s}\right)$ at $y=y_{s}=y_{c}$. At such a point one has $S=T=U$ or $H_{U}^{2}\left(y_{c}\right)$ $=4 H_{S}^{2}\left(y_{c}\right)=4 H_{T}^{2}\left(y_{c}\right)$. In terms of the parameters in the harmonic function this means that one must arrange $\left(c_{U}-c_{T}-c_{S}\right) /\left(d_{U}-d_{T}-d_{S}\right)=\left(c_{T}-c_{S}\right) /\left(d_{T}-d_{S}\right) \Leftrightarrow\left|y_{c}\right|=\left|y_{s}\right|$. Generically this condition is not satisfied and therefore $\left|y_{c}\right|<\left|y_{s}\right|$.

Now we can set up an example of a solution running into the enhancement boundary. We take care of the constraint $H_{T}>H_{S}$ by setting $H_{T}=2 H_{S}$. It will turn out that this will lead to relatively simple analytic expressions. Note that this choice implies that at the enhancement boundary $U$ $=T$ one has $S=2 U$ or in terms of $h^{I}$ : at $h^{2}=0$ one has $h^{1}=h^{3}$, (see Fig. 2).

The harmonic functions take the form $H_{I}=c_{I}-d_{I}|y|$ dictated by the presence of two spacetime boundaries. The constants $c_{I}$ define the initial condition on the first space-time boundary whereas the slopes $d_{I}$ determine how the solution flows through moduli space. The $c_{I}$ are undetermined integration constants, which are only restricted by the fact that all scalars should be inside the Kähler cone at $y=0$ and by the conventional normalization $a=1$ that we impose on the metric at $y=0$. On the other hand, the $d_{I}$ are, in the context of a Calabi-Yau compactification with flux, determined by the sources of flux put on the boundaries. ${ }^{4,15} \mathrm{We}$ will choose some values for $d_{I}$ to simplify the calculations and not try to connect these values to particular sources of fluxes, 


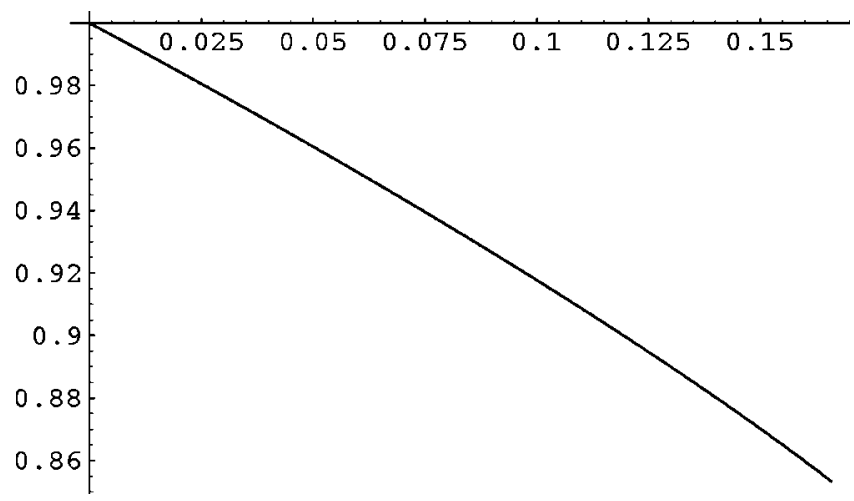

FIG. 1. The function $a(y)$ for $0 \leqslant y \leqslant 0.17$.

since we have shown that the picture is generic. It will not change when taking different slopes, as long as the solution runs into the enhancement boundary without reaching any other boundary of moduli space first.

Now we choose initial data. We have $c_{T}=2 c_{S}$ and have to impose $c_{U}>c_{T}+c_{S}=3 c_{S}$. For definiteness we take $c_{U}=4 c_{S}$. Then $c_{S}$ is fixed by the normalization condition $a(0)=1$. This can be solved exactly with the result

$$
c_{S}=\left(\frac{45}{49}+\frac{9}{49 \sqrt{2}}\right)^{1 / 3} .
$$

Now we have to set the slope. We already decided to take $d_{T}=2 d_{S}$. Then the boundary $T=U$ is reached once the inequality

$$
|y| \leqslant\left|y_{e}\right|=\frac{C_{U}-c_{T}-c_{S}}{d_{U}-d_{T}-d_{S}}=\frac{c_{S}}{d_{U}-3 d_{S}}
$$

is saturated. We are free to choose $d_{U}>3 d_{S}$. For definiteness we take $d_{U}=10$ and $d_{S}=1$. The analytical value of $\left|y_{e}\right|$ is

$$
\left|y_{e}\right|=\frac{1}{7}\left(\frac{45}{49}+\frac{9}{49 \sqrt{2}}\right)^{1 / 3} \simeq 0.145118
$$

Then $a(|y|)$ is well behaved for $0 \leqslant|y| \leqslant\left|y_{e}\right|$. However, $a^{3}$ becomes complex and the scalar curvature $\mathcal{R}$ becomes infinite for some $\left|y_{s}\right|>\left|y_{e}\right|$. Looking at the explicit analytic expressions for $a$ and $\mathcal{R}$ one sees that this happens, independently of our concrete choice of parameters, because $\sqrt{H_{U}^{2}-4 H_{S} H_{T}}$ vanishes and then becomes complex.

In our concrete numerical example the equation $H_{U}^{2}-4 H_{S} H_{T}=0$ has two roots, the relevant being

$$
\left|y_{s}\right|=\frac{1}{23}\left(8\left(\frac{45}{49}+\frac{9}{49 \sqrt{2}}\right)\right)^{1 / 3}-3 \sqrt{2}\left(\frac{45}{49}+\frac{9}{49 \sqrt{2}}\right)^{1 / 3} \simeq 0.165949,
$$

such that indeed $\left|y_{s}\right|>\left|y_{e}\right|$. As we explained earlier, this holds generically for solutions running into the direction of the enhancement boundary. Whenever the solution runs into the specific boundary of moduli space, where gauge symmetry is enhanced, then it reaches this boundary before the space-time curvature becomes infinite. This is an example where a moduli space boundary shields a space-time singularity. 


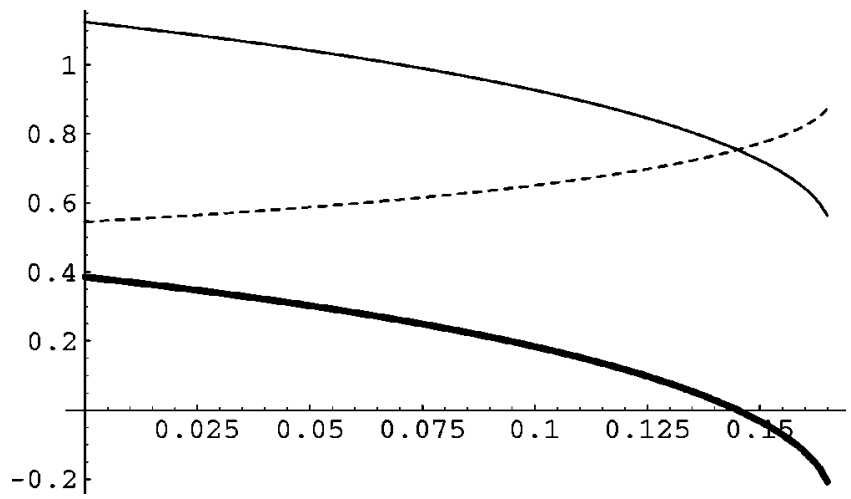

FIG. 2. The Kähler moduli $h^{1}(y)$ (dashed line), $h^{2}(y)$ (thick line), and $h^{3}(y)$ (thin line) for $0 \leqslant y \leqslant 0.17$. At $y=y_{c}$ $\cong 0.145118$ the four-cycle associated to $h^{2}$ has collapsed. Note that this happens before the space-time singularity occurs at $y=y_{s} \cong 0.165949$.

The analytical values of $a, \mathcal{R}$ at $\left|y_{e}\right|$ in the example are

$$
a(|\tilde{y}|)=\left(\frac{3}{7}\left(\frac{45}{49}+\frac{9}{49 \sqrt{2}}\right)\right)^{1 / 6} \quad \text { and } \quad \mathcal{R}(|\tilde{y}|)=\frac{7}{3}\left(\frac{7}{3}\right)^{1 / 3} \text {. }
$$

The analytical expressions of $a, \mathcal{R}$ at $\left|y_{s}\right|$ for our example are complicated and therefore we do not display them. It is, however, instructive to plot various quantities for our specific set of parameters.

We display the metric $a$ in Fig. 1, the moduli $h^{1}, h^{2}, h^{3}$ which solve the generalized attractor equation in Fig. 2, and the space-time curvature $\mathcal{R}$ in Fig. 3 for $0 \leqslant y \leqslant 0.17$. Clearly, the cycle $h^{2}$ collapses at $\left|y_{c}\right|=\left|y_{e}\right| \simeq 0.145118 \ldots$. At this point the space-time is perfectly regular! Further down at $\left|y_{s}\right| \simeq 0.165949 \ldots$, where the cycle $h^{2} \simeq-0.2527 \ldots$ is already negative, i.e., unphysical, the space-time has a naked singularity. All this follows from the solution of the Einstein equation in the bulk under the condition that we have not yet put the second wall at some $|\tilde{y}|$.

Note that the GST and HW models show the same qualitative behavior. This is as expected because the singular behavior is due to singularities in the function $a$ and its derivatives.

From the point of view of supergravity nothing special happens along the line $T=U$ in the scalar manifold. A negative value of the scalar field $h^{2}=T-U$ is as good as a positive one since the metric on the moduli space at $h^{2}=0$ is regular and there is no reason to consider $T=U$ as a boundary. According to supergravity one can continue the solution to negative $T-U$ and finally

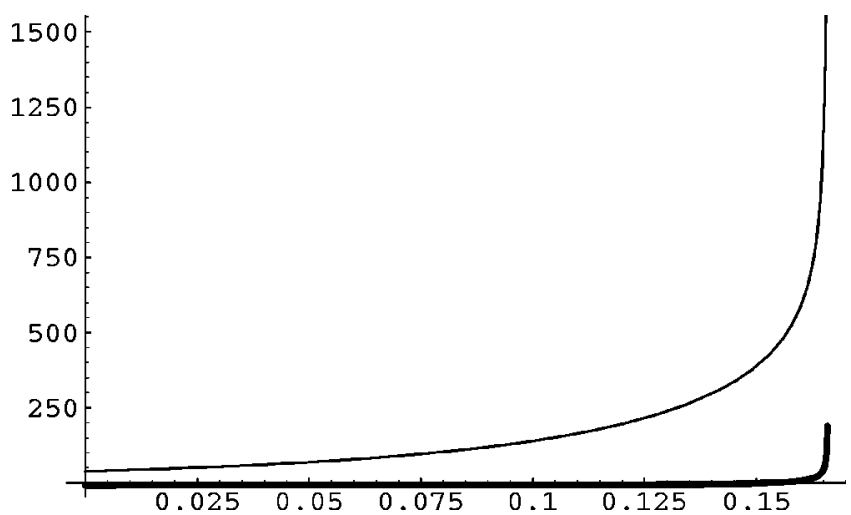

FIG. 3. The Ricci scalar $R(y)$ for $0 \leqslant y \leqslant 0.17$. The thick line refers to the GST model, the thin line to the HW model. In both cases the Ricci scalar diverges at $y=y_{s} \cong 0.165949$. 
one encounters a space-time singularity at $|y|=\left|y_{s}\right|$. In order to avoid the singularity one has to put the second brane at some place $|\tilde{y}|<\left|y_{s}\right|$, but there is no distinguished choice of such $|\tilde{y}|$, nor a physical mechanism which excises the singularity. Since our solution is supersymmetric, it has zero energy, as shown in Ref. 2, independently of the position of the second brane.

This is different in M/string theory. In M-theory compactified on CY three-folds $h^{2}$ must be positive as a volume of the cycle. $T=U$ is a line of $\mathrm{SU}(2)$ gauge symmetry enhancement and $T$ $-U$ is the associated Higgs field. Therefore the moduli space ends at $T=U$, and negative values of $T-U$ are related to positive values by the action of the generator of the Weyl group of SU(2), which is isomorphic to $\mathbf{Z}_{2}$. This takes a particularly familiar form when using the dual heterotic description, where $R:=\sqrt{T / U}$ is the radius of the sixth dimension. Therefore the Weyl twist acts as T-duality $R \rightarrow 1 / R$ and $\mathrm{SU}(2)$ gauge symmetry enhancement occurs at the self-dual radius, $R$ $=1{ }^{24,25}$ Since the gauge symmetry enhancement happens at $y_{e}<y_{s}$ it does not make sense to naively continue to $y>y_{e}$ and in this way the singularity is excised. Moreover, it may be natural to put the second brane precisely at the enhançon point $y=y_{e}$, defined by the equation

$$
R\left(y_{e}\right)=1, \quad R^{2}(y) \equiv \frac{T(y)}{U(y)}=\frac{h^{1}+h^{2}}{h^{1}} .
$$

In this case the $\mathbf{Z}_{2}$ orbifold symmetry is acting on $y$ and the Weyl twist/T-duality transformation is acting on the moduli coincide. By putting the second wall at the enhançon point $y=y_{e}$ we enforce the physics to depend on $|T-U|$. When putting the second wall at a different place we would break T-duality spontaneously.

So far we have worked with the prepotential $S T U+1 / 3 U^{3}$ valid inside the Kähler cone. We found that both the resulting theory and the domain wall solutions were regular at $T=U$. However, we had to stop there because we reached a boundary and new physics occurred. One way to capture this new physics is to use the $T \leftrightarrow U$ symmetric form of the prepotential that was found in Ref. 26 in the context of heterotic string theory on $K 3 \times S^{1}$ :

$$
\mathcal{V}=S T U+\frac{1}{3} U^{3} \theta(T-U)+\frac{1}{3} T^{3} \theta(U-T) .
$$

This is now valid for both positive and negative $T-U$. The build-in $T \leftrightarrow U$ symmetry reflects that negative $T-U$ is related to positive $T-U$ by a large gauge transformation. The resulting discontinuities are consequences of the $\mathrm{SU}(2)$ gauge symmetry enhancement and reflect the presence of extra massless states at $T=U$. They are analogs of the logarithmic branch cuts one encounters in four dimensions. ${ }^{26,27}$

Earlier we mentioned that it may be natural to put the second wall at the enhançon locus so that $|\tilde{y}|=\left|y_{e}\right|$. We can use the heterotic prepotential (34) to give an additional argument for this. Namely, the presence of the discontinuities in the prepotential automatically causes a $\delta$-function singularity in the space-time geometry of a domain wall which tries to cross the boundary $T$ $=U$. Therefore the enhançon itself acts like a source. Note that this kink singularity is different from the naked singularities of the supergravity solution that we want to excise.

To see this explicitly we first recall that singularities of the Ricci scalar come from singularities of $a^{\prime \prime}$, where ' is the derivative with respect to $y$ and $a(y)=(\mathcal{V}(\widetilde{h}(y)))^{1 / 3}$. Singularities in $a^{\prime \prime}$ can therefore descend from the $\theta$-functions which are present in (34) through application of the chain rule. To work this out we need to be more precise about how $\mathcal{V}$ behaves as a function of $\widetilde{T}-\widetilde{U}$. Despite the presence of the $\theta$-functions, $\mathcal{V}$ itself is actually continuous, but its derivative with respect to $\widetilde{T}-\widetilde{U}$ has a finite jump at $\widetilde{T}=\widetilde{U}$. Consequently the second derivative gives a $\delta$-function: $\partial^{2} \mathcal{V} / \partial(\widetilde{T}-\widetilde{U}) \partial(\widetilde{T}-\widetilde{U})=-((\widetilde{T}+\widetilde{U}) / 2)^{2} \delta(\widetilde{T}-\widetilde{U})+$ finite. This contributes to $a^{\prime \prime}: a^{\prime \prime}$ $=1 / 3 \mathcal{V}(\widetilde{h})^{-2 / 3} \mathcal{V}^{\prime \prime}+$ finite $\simeq\left[\partial^{2} \mathcal{V} / \partial(\widetilde{T}-\widetilde{U}) \partial(\widetilde{T}-\widetilde{U})\right]\left[(\widetilde{T}-\widetilde{U})^{\prime}\right]^{2}+$ finite, where we dropped terms, both additive and multiplicative, that stay finite for $\widetilde{T}=\widetilde{U}$. Since $\widetilde{T}-\widetilde{U}$ has $y=y_{e}$ as its only zero we find 


$$
a^{\prime \prime} \sim-\left(\frac{\widetilde{T}+\widetilde{U}}{2}\right)^{2}(\widetilde{T}-\widetilde{U})^{\prime} \delta\left(y-y_{e}\right)
$$

This would justify our assertion that it is natural to put the second wall at the enhancement point so that $|\tilde{y}|=\left|y_{e}\right|$. Any other position will break the T-duality symmetry.

By five-dimensional heterotic-M-theory duality we expect that the physics of SU(2) enhancement can be equivalently described in the M-theory language. In the context of Calabi-Yau compactifications $\mathrm{SU}(2)$ gauge symmetry enhancement (with $g \geqslant 0$ additional hypermultiplets) occurs when a divisor collapses into a (genus $g$ ) curve of $A_{1}$ singularities. In our case we know from the heterotic analysis that this curve must have genus 0 . The Weyl group $\mathbf{Z}_{2}$ is encoded in the geometry through the local form of the $A_{1}$ singularity, $\mathbf{C}^{2} / \mathbf{Z}_{2}$. It seems that the Weyl reflections relating positive to negative $T-U$ in the heterotic language correspond to the "elementary transformations" discussed in Ref. 28. The extension of the range of moduli as done in (34) presumably corresponds to the procedure of gluing in a reflected Kähler cone at the enhancement boundary, which is described in Ref. 28.

In this article we have shown that there is a stringy mechanism which in certain cases excises space-time singularities which plague supergravity solutions. The mechanism is based on the fact that the stringy moduli space has a boundary on which the moduli space metric is finite. Whereas this boundary does not have a particular meaning in supergravity, so that solutions can be continued beyond until a space-time singularity occurs, one encounters new physics at the boundary in string theory, which makes the space-time singularity unphysical.

This observation leads to a variety of new issues which have to be addressed in the future. Most importantly one would like to understand in detail how the new M/string theory physics modifies space-time geometry and excises the singularity. Since SU(2) gauge symmetry enhancement occurs at the boundary, the situation resembles the enhançon geometry ${ }^{12}$ and it would be interesting to explore how far this parallel goes. There are some further facts which might be relevant. In particular at the boundary the tensionless magnetic strings are present in addition to charged massless gauge bosons: it was shown in Ref. 9 that the magnetic string states with charges $\pm(1,-2,1)$ have a vanishing tension. Also one should take into account that the five-dimensional prepotential is purely cubic for five noncompact dimensions. However, in our domain wall setup the fifth dimension is compact and subject to an orbifold projection which reduces the number of unbroken supersymmetries. Thus the new stringy physics at the boundary might be more complex and more interesting than naively expected.

We are very grateful to I. Antoniadis, S. Dimopoulos, S. Kachru, A. Linde, E. Silverstein, L. Susskind, and N. Toumbas for useful discussions. T.M. would like to thank Y. Zunger for help in using Mathematica. This work was supported by NSF Grant No. PHY-9870115.

${ }^{1}$ M. Günaydin, G. Sierra, and P. K. Townsend, Nucl. Phys. B 253, 573 (1985).

${ }^{2}$ E. Bergshoeff, R. Kallosh, and A. Van Proeyen, J. High Energy Phys. 0010, 033 (2000) hep-th/0007044.

${ }^{3}$ The study of collapsing CY cycles was reported recently in Ref. 4.

${ }^{4}$ B. R. Greene, K. Schalm and G. Shiu, hep-th/0010207.

${ }^{5}$ E. Witten, Nucl. Phys. B 471, 135 (1996) hep-th/9602070.

${ }^{6}$ A. Lukas, B. A. Ovrut, K. S. Stelle, and D. Waldram, Nucl. Phys. B 552, 246 (1999) hep-th/9806051.

${ }^{7}$ P. Hořava and E. Witten, Nucl. Phys. B 475, 94 (1996) hep-th/9603142.

${ }^{8}$ S. Ferrara, R. Kallosh, and A. Strominger, Phys. Rev. D 52, 5412 (1995) hep-th/9508072; A. Strominger, Phys. Lett. B 383, 39 (1996) hep-th/9602111; S. Ferrara and R. Kallosh, Phys. Rev. D 54, 1514 (1996) hep-th/9602136; Phys. Rev. D 54, 1525 (1996) hep-th/9603090; S. Ferrara, G. W. Gibbons, and R. Kallosh, Nucl. Phys. B 500, 75 (1997) hep-th/9702103; G. Moore, hep-th/9807087; F. Denef, Nucl. Phys. B 547, 201 (1999) hep-th/9812049.

${ }^{9}$ A. Chou, R. Kallosh, J. Rahmfeld, S.-J. Rey, M. Shmakova, and W. K. Wong, Nucl. Phys. B 508, 147 (1997) hep-th/9704142.

${ }^{10}$ W. A. Sabra, Mod. Phys. Lett. A 13, 239 (1998) hep-th/9708103.

${ }^{11}$ I. Gaida, S. Mahapatra, T. Mohaupt, and W. A. Sabra, Class. Quantum Grav. 16, 419 (1999) hep-th/9807014.

${ }^{12}$ C. V. Johnson, A. W. Peet, and J. Polchinski, Phys. Rev. D 61, 086001 (2000) hep-th/9911161, C. V. Johnson, hep-th/0004068

${ }^{13}$ N. Arkani-Hamed, S. Dimopoulos, N. Kaloper, and R. Sundrum, Phys. Lett. B 480, 193 (2000) hep-th/0001197; S. Kachru, M. Schulz, and E. Silverstein, Phys. Rev. D 62, 045021 (2000) hep-th/0001206; M. Cvetic, H. Lu, and C. N. 
Pope, hep-th/0002054; H. Verlinde, hep-th/0004003; M. J. Duff, J. T. Liu, and K. S. Stelle, hep-th/0007120.

${ }^{14}$ E. Witten, Nucl. Phys. B 471, 195 (1996) hep-th/9603150.

${ }^{15}$ K. Behrndt and S. Gukov, Nucl. Phys. B 580, 225 (2000) hep-th/0001082.

${ }^{16} \mathrm{We}$ use the notation and conventions of Ref. 2.

${ }^{17}$ P. Candelas, A. Font, S. Katz, and D. R. Morrison, Nucl. Phys. B 429, 626 (1994) hep-th/9403187; D. R. Morrison and C. Vafa, Nucl. Phys. B 473, 74 (1996) hep-th/9602114; Nucl. Phys. B 476, 437 (1996) hep-th/9603161.

${ }^{18}$ J. Louis, J. Sonnenschein, S. Theisen, and S. Yankielowicz, Nucl. Phys. B 480, 185 (1996) hep-th/9606049.

${ }^{19}$ Recently an extensive study of the flop transition in the context of supersymmetric domain walls was performed in Ref.

${ }^{20}$ The space-time curvature may still be finite if $\left(\phi^{x}\right)^{\prime}, a^{-2 \gamma}, W_{, x}$ vanish at the singularity of the moduli space.

${ }^{21}$ The harmonic functions $H_{I}=c_{I}-d_{I}|y|$ used here and below are different from the harmonic functions in Ref. 2 and shown in Eq. (8) by a factor of 6 . The first term $c_{I}$ is arbitrary, so we will call it again $c_{I}$, the second term is $d_{I}$ $=1 / 3 g q_{I}$. This normalization of the harmonic functions differs by a factor of 2 from the one used in Ref. 11 and more recently in Ref. 4. When comparing to the solution of the attractor equations given in Ref. 9 one should also rescale the charges.

${ }^{22}$ The divergences in $a^{\prime}$ cancel (for the generic case where $a \neq 0$ ) as required by the relation $a \dot{a} \sim W$ implied by very special geometry [see equation (6.19) in Ref. 2]. However, $a^{\prime \prime}$ is generically singular when $U=0$.

${ }^{23}$ I. Gaida, Phys. Lett. B 429, 297 (1998) hep-th/9802140.

${ }^{24}$ R. Brandenberger and C. Vafa, Nucl. Phys. B 316, 391 (1989); A. A. Tseytlin and C. Vafa, ibid. 372, 443 (1992) hep-th/9109048.

${ }^{25}$ The situation reminds us of some earlier suggestions (Ref. 24) to remove the Big Bang singularity using $R \rightarrow 1 / R$ symmetry of the string theory.

${ }^{26}$ I. Antoniadis, S. Ferrara, and T. R. Taylor, Nucl. Phys. B 460, 460 (1996) hep-th/9511108.

${ }^{27}$ This applies equally to the $T=U$ line in the $(12,12),(13,11)$, and $(14,10)$ model. Note that the new physics at the other boundaries is not taken care of.

${ }^{28}$ S. Katz, D. R. Morrison, and M. Ronen Plesser, Nucl. Phys. B 477, 105 (1996) hep-th/9601108. 